\title{
Controle de qualidade físico-químico e caracterização fitoquímica das principais plantas medicinais comercializadas na feira-livre de Lagarto-SE
}

\author{
Physical-chemical quality control and phytochemical characterization of the main medicinal \\ plants marketed in fair-free of Lagarto-SE \\ C. A. S. Souza ${ }^{1 *}$; L. N. Almeida ${ }^{2}$; E. S. Cruz ${ }^{2}$; C. M. L. Silva²; J. A. C. \\ Nascimento Júnior ${ }^{2}$; F. S. Amaral ${ }^{1} ;$ M. R. Serafini ${ }^{2}$ \\ ${ }^{1}$ Laboratório de Farmacognosia, Departamento de Farmácia, Universidade Federal de Sergipe, 49000-000, São \\ Cristovão-Sergipe, Brasil. \\ ${ }^{2}$ Laboratório de Ensaiso Farmacêuticos e Toxicidade, Departamento de Farmácia, Universidade Federal de Sergipe, \\ 49000-000, São Cristovão-Sergipe, Brasil.
}

*carlos.953@gmail.com

(Recebido em 19 de dezembro de 2016; aceito em 24 de setembro de 2017)

\begin{abstract}
Segundo a Organização Mundial de Saúde (OMS), as plantas medicinais contribuem para a promoção da saúde, prevenção de doenças reabilitação e cura. Neste contexto, o presente estudo buscou realizar a caracterização físico - químico e fitoquímico das plantas medicinais mais comercializadas na feira - livre de Lagarto, munícipio do estado de Sergipe. As plantas medicinais mais comercializadas foram Plectranthus barbatus Andrews (boldo) 40\%, Lippia alba (Mill.) N. E. Brown (erva cidreira) 25\% e Pimpinella anisum L. (erva doce) 20\%. Foi possível constatar os grupos dos compostos químicos provenientes do metabolismo secundário das plantas de cada espécie estudada com a ausência de alguns compostos descritos na literatura. Com relação a caracterização fitoquímica a espécie vegetal do boldo estava em sua maior parte dentro dos padrões estabelecidos pela farmacopeia, contudo as outras plantas medicinais ainda não estão incluídas na farmacopeia. Os testes realizados são vitais para o controle de qualidade, proporcionando segurança para os usuários, além de fornecer dados para futuros estudos para a padronização e desenvolvimento de novos fármacos.

Palavras-chave: Plantas medicinais; Controle de qualidade; Fitoterapia; Fitoquímica; Físico-química.
\end{abstract}

According to the World Health Organization (WHO), as medicinal plants contribute to health promotion, disease prevention, rehabilitation and healing. In this context, the present study sought to perform a physical - chemical and phytochemical characterization of the most commercialized medicinal plants in the free trade fair of Lagarto, municipality of Sergipe state. The most commercialized medicinal plants were Plectranthus barbatus Andrews 40\%, Lippia alba (Mill.) N. E. Brown 25\% and Pimpinella anisum L. 20\%. It was possible groups of chemicals from the secondary metabolism of plants of each species studied in the absence of some groups described in the literature. Regarding the characterization phytochemical plant species Plectranthus barbatus Andrews was mostly within the standards set by the pharmacopoeia, but other medicinal plants are not yet standardized in the pharmacopoeia. The tests are vital for quality control, providing security for the users, and provide data for future studies to the standardization and development of new drugs.

Keywords: Medicinal Plants; Quality control; Phytotherapy; Phytochemistry; Physicochemical.

\section{INTRODUÇÃO}

A Organização Mundial da Saúde (OMS) relata a importância do uso de plantas medicinais no ambiente de saúde, tendo ciência de que $80 \%$ da população dos países em desenvolvimento fazem uso de plantas medicinais ou preparações destas no que se refere à atenção primária de saúde [1].

No Brasil em 2006, o Ministério da Saúde criou a Política Nacional de Práticas Integrativas e Complementares (PNPIC) introduzida no âmbito do Sistema Único de Saúde (SUS) com o propósito de expandir as alternativas terapêuticas oferecidas aos usuários, com garantia de acesso as plantas medicinais, fitoterápicos e outras práticas relacionadas, com segurança, eficácia e qualidade [2]. Entretanto, o uso seguro de plantas medicinais no Brasil ainda é desejável, devido a necessidade de 
maior conhecimento sobre as propriedades químicas, farmacológicas e toxicológicas, estabelecendo critérios de eficácia, segurança e qualidade para a população.

Neste contexto, a realização de testes físico químicos e fitoquímicos são essenciais para o controle de qualidade de plantas medicinais, no intuito de proporcionar a segurança e a efetividade. A análise do teor dos principais componentes biologicamente ativos em matérias primas de origem vegetal ou fitoterápicos é essencial para garantir a autenticidade, pureza e integridade das ervas medicinais. Os testes fitoquímicos procuram evidenciar as principais classes de metabólitos através do uso de técnicas cromatográficas e analíticas que permitam a separação e o isolamento de substâncias para o conhecimento da composição química, como também para a padronização do material vegetal e produtos relacionados [3].

Diante do exposto, o objetivo desta pesquisa, foi caracterizar por meio de testes físico - químico e fitoquímicos as plantas medicinais Plectranthus barbatus Andrews (boldo), Lippia alba (Mill.) N. E. Brown (erva cidreira) e Pimpinella anisum L. (erva doce) principais plantas medicinais comercializadas na feira livre do munícipio de Lagarto.

\section{MATERIAL E MÉTODOS}

Desenho do estudo

Trata-se de um estudo transversal, prospectivo realizado na feira livre do município de Lagarto SE, no período de setembro a outubro de 2015. Os critérios de inclusão para participar do estudo foram: Comerciantes de plantas medicinais, maiores de 18 anos, que aceitaram participar do estudo e assinaram o termo de consentimento livre e esclarecido.

Caracterização da área de estudo

O município está localizado na região Centro - Sul do estado de Sergipe, distante 78 quilômetros da capital Aracaju, sua população em 2010 era composta por 94.861 habitantes, segundo dados do Instituto Brasileiro de Geografia e Estatística (IBGE) [4].

Aplicação de questionário

A escolha das plantas medicinais para a pesquisa, foi realizada por meio de um questionário estruturado entre os feirantes com o intuito de descobrir quais plantas medicinais eram mais comercializadas. A amostra foi selecionada por conveniência (30 comerciantes) e o questionário adaptado de Silva et al. (2013) [5] incluía questões que abordavam dados sócio econômicos, estado de saúde e plantas medicinais utilizadas pelos comerciantes.

Coleta das plantas medicinais

Foram coletadas as plantas medicinais com maior índice de comercialização na feira livre do município de Lagarto - SE. As plantas medicinais foram recolhidas na feira livre seguindo os protocolos botânicos de coleta de espécies vegetais. O procedimento para a coleta foi o reconhecimento da área, coleta das plantas medicinais, preparação da exsicata e registro da atividade. Para tal fim, foi realizada uma investigação sobre quais feirantes vendiam e indicavam ervas medicinais. A coleta das informações das plantas medicinais cultivadas pelos comerciantes foi através do método da bola de neve "snow ball sampling".

Esta é uma técnica de amostragem não probabilística utilizada na pesquisa social, na qual os participantes iniciais da pesquisa indicam novos participantes que por sua vez indicam novos participantes e assim sucessivamente até que seja alcançado o objetivo proposto (o "ponto de saturação"). O "ponto de saturação" é atingido quando novos entrevistados passam a repetir os conteúdos já obtidos em entrevistas anteriores, sem acrescentar novas informações relevantes a pesquisa [6]. 
Posteriormente as espécies selecionadas foram identificadas pelo herbário do Departamento de Biologia da Universidade Federal de Sergipe, com posterior depósito de exsicata. Com os resultados obtidos foram realizados testes fitoquímicos e controle de qualidade das seguintes plantas: $P$. barbatus (boldo), L. alba (erva cidreira) e P. anisum (erva doce).

Aspectos éticos

O presente trabalho foi submetido ao Comitê de Ética e Pesquisa do Hospital Universitário da Universidade Federal de Sergipe. Assim, todos os participantes que concordaram em participar do estudo foram esclarecidos quanto às metas e a natureza da pesquisa, assinando um termo de consentimento livre e esclarecido, de acordo com a Resolução CNS n ${ }^{\circ} 466 / 12$ [7]. A presente pesquisa foi aprovada pelo Comité de Ética em Pesquisa Envolvendo Humanos (CEP-UFS) sob o número de protocolo 50133615.3.0000.

Triagem Fitoquímica

Para a realização dos testes fitoquímicos foi seguida a metodologia proposta por Matos (1997) [8]. Foi realizada a triagem fitoquimica para a identificação de fenóis, taninos, saponinas, alcalóides, catequinas e flavonas, antocianinas, antocianidas e flavanóides, chaconas e auronas, flavanóis, flavanonas, flavanonóis e xantonas, esteroides e triterpernóides.

Controle de Qualidade

Os testes de caracterização físico-químico foram realizados para determinar: densidade, $\mathrm{pH}$, resíduo seco, perda por dessecação e teor de cinzas totais, tudo conforme estabelecido pela Farmacopeia Brasileira V (2010) [9].

a) Processamento das amostras

Após a coleta, as drogas vegetais frescas (plantas inteiras), foram secas em estufa por 2 horas a $105^{\circ} \mathrm{C}$. Realizada a secagem das espécies vegetais as plantas foram trituradas em moinho de facas, obtendo-se pós finos secos.

b) Determinações de perda por dessecação e de cinzas totais

A determinação da perda por dessecação (teor de umidade) do pó do P. barbatus, L. alba e $P$. anisum foi realizada através do método gravimétrico, descrito na Farmacopeia Brasileira V (2010) [9]. A determinação quantitativa do resíduo não volátil (cinzas totais) do pó das amostras foi realizada também de acordo com o método descrito na Farmacopeia Brasileira V (2010) [9].

c) Obtenção e caracterização físico-química das soluções extrativas hidroalcoólicas

O método escolhido para a obtenção dos extratos $P$. barbatus, $L$. alba e $P$. anisum foi a percolação utilizando as folhas secas das plantas.

d) Determinação da densidade e do pH

Foram determinados a densidade e o pH de acordo com a Farmacopéia Brasileira V (2010) [9]. Para a determinação potenciométrica do $\mathrm{pH}$ das soluções extrativas hidroalcoólicas do $P$. barbatus, $L$. $a l b a$ e $P$. anisum foi feita pela medida da diferença de potencial entre dois eletrodos adequados, imersos na solução em teste. Um destes eletrodos é sensível aos íons hidrogênio e o outro é o eletrodo de referência, de potencial constante.

A densidade das soluções extrativas hidroalcoólicas do P. barbatus, L. alba e P. anisum foi feita em um picnômetro. Este instrumento refere-se a um pequeno frasco de vidro que possibilita que o volume do fluido acomodado seja invariável. Apresentando uma larga abertura e tampa perfurada na forma de um fino tubo em sentido longitudinal.

e) Determinação da porcentagem de resíduo seco total 
Exatamente $20 \mathrm{~mL}$ de cada extrato foram pipetados e transferidos para béqueres previamente tarados. Os béqueres foram colocados na chapa aquecedora até secura e, em seguida, levados à estufa a $105^{\circ} \mathrm{C}$, até obter peso constante. Após resfriarem em dessecador, os béqueres foram pesados e calculadas as percentagens de resíduo seco.

\section{RESULTADOS E DISCUSSÃO}

Foram entrevistados 30 comerciantes, destas $83,3 \%$ eram do sexo feminino. A média de idade dos participantes foi de 49,6 anos ( $\pm 13,10)$. Com relação ao nível de escolaridade $90 \%$ sabem ler e escrever, contudo a maioria dos entrevistados $53,3 \%$ apresentavam no máximo até quatro anos de estudo. Entre as plantas medicinais mais comercializadas destacam-se o P. barbatus (40\%), L. alba (25\%) e P. anisum (20\%) e outras espécies (15\%). Esses resultados são similares a outro estudo realizado no mesmo munícipio que relatam entre as plantas utilizadas a $L$. alba e a $P$. anisum pelos pacientes oncológicos [10].

No que concerne as partes das plantas utilizadas e citadas como indicação de uso pelos comerciantes, destacam-se as folhas $58,82 \%$, seguido da planta seca armazenada em saco plástico $19,61 \%$, sementes $9,80 \%$, flores $5,88 \%$, casca 3,92\% e frutos 1,96\% (Figura 1). Com relação ao uso e indicação das formas extrativas indicadas pelos comerciantes destacam-se a infusão $52,5 \%$ e a decocção 32,5\%. Nesse estudo houve relatos variados quanto ao uso das folhas, com a maior parte indicando a infusão, seguido da decocção. Segundo Júnior Veiga et al. (2005) [11], a depender da parte da planta utilizada, as formas de preparo dos remédios caseiros também variam, portanto, é necessário utilizar a parte vegetal de maneira adequada, possibilitando a correta extração dos princípios ativos e, consequentemente, a manutenção do efeito farmacológico.

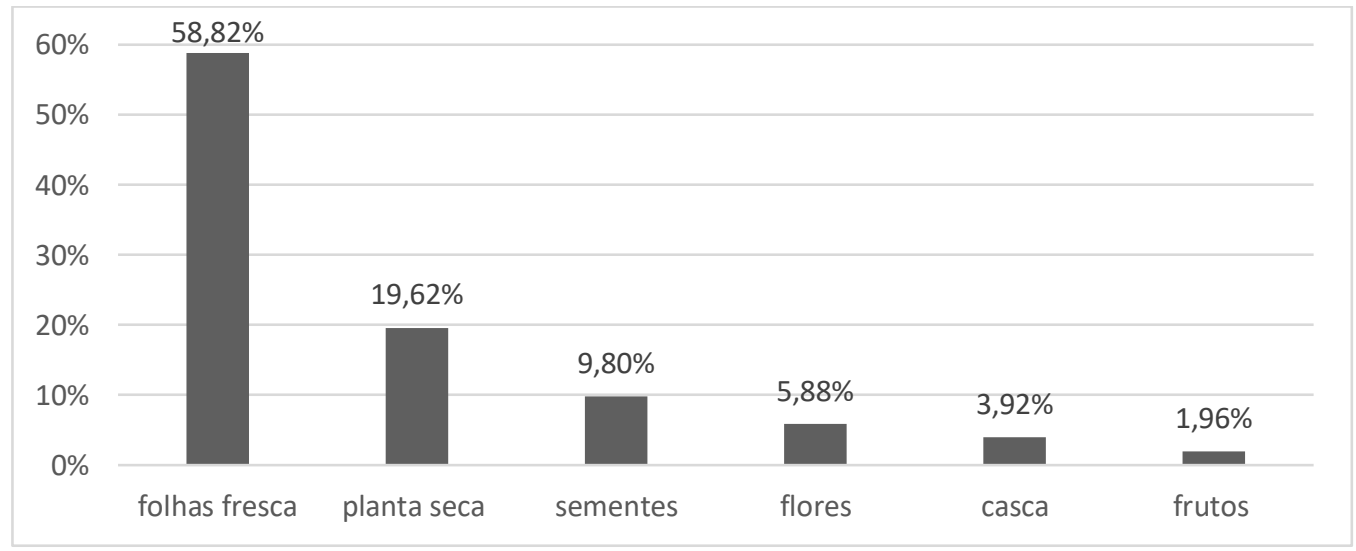

Figura 1. Percentual das partes das plantas indicadas pelos comerciantes para tratamento de doenças.

Com a realização da caracterização fitoquímica foi possível constatar os grupos dos compostos químicos provenientes do metabolismo secundário das plantas (Fenóis e Taninos, Saponinas, Alcalóides, Catequinas e Flavonas, Antocianinas, Antocianidinas e Flavanóides, Chaconas e Auronas, Flavonóis, Flavanonas, flavanonóis e xantonas e Esteróides e Triterpenóides). Para a confirmação ou não da presença de metabólitos secundários nas espécies avaliadas neste trabalho, foram utilizadas reações avaliando determinada característica esperada ou não, indicando assim a sua presença ou ausência. A Tabela 1 mostra os resultados obtidos após a realização da triagem fitoquímica. 
Tabela 1: Triagem Fitoquímica dos extratos hidroalcoólicos das folhas de Plectranthus barbatus, Lippia alba e Pimpinella anisum.

\begin{tabular}{|c|c|c|c|c|}
\hline \multirow[b]{2}{*}{$\begin{array}{r}\text { Classe de } \\
\text { compostos }\end{array}$} & \multirow[b]{2}{*}{ Reações } & \multicolumn{3}{|c|}{ Espécies vegetais e seus extratos } \\
\hline & & $\begin{array}{c}\text { Plectranthus } \\
\text { barbatus A. }\end{array}$ & $\begin{array}{r}\text { Lippia } \\
\text { alba } \mathrm{M} .\end{array}$ & $\begin{array}{c}\text { Pimpinella } \\
\text { anisum L. }\end{array}$ \\
\hline $\begin{array}{l}\text { Fenóis e } \\
\text { Taninos }\end{array}$ & Cloreto Férrico & + & - & + \\
\hline Saponinas & Índice de Espuma & + & - & - \\
\hline & Hager & - & - & - \\
\hline Alcalóides & Mayer & - & - & - \\
\hline & Dragendorff & + & - & - \\
\hline $\begin{array}{r}\text { Catequinas } \\
\text { Flavonas }\end{array}$ & $\begin{array}{l}\text { Acidulação e } \\
\text { Alcalinização }\end{array}$ & + & - & - \\
\hline $\begin{array}{r}\text { Antocianinas, } \\
\text { Antocianidinas e } \\
\text { Flavanóides }\end{array}$ & $\begin{array}{l}\text { Acidulação e } \\
\text { Alcalinização }\end{array}$ & + & - & - \\
\hline $\begin{array}{r}\text { Chaconas e } \\
\text { Auronas }\end{array}$ & $\begin{array}{l}\text { Acidulação e } \\
\text { Alcalinização }\end{array}$ & + & - & - \\
\hline Flavonóis & $\begin{array}{l}\text { Acidulação e } \\
\text { Alcalinização }\end{array}$ & + & - & - \\
\hline $\begin{array}{r}\text { Flavanonas, } \\
\text { Flavanonóis e } \\
\text { Xantonas }\end{array}$ & $\begin{array}{l}\text { Acidulação e } \\
\text { Alcalinização }\end{array}$ & - & + & + \\
\hline $\begin{array}{r}\text { Esteróides e } \\
\text { Triterpenóides }\end{array}$ & Liebermann-Buchard & + & - & - \\
\hline
\end{tabular}

A constatação das reações de caracterização fitoquímica ocorreu pelas seguintes respostas: coloração variável entre o azul e o vermelho confirmou a presença de fenóis e taninos após a adição da solução alcoólica de $\mathrm{FeCl}_{3}$. No teste para identificação de saponinas houve formação de espuma persistente e abundante caracterizando teste positivo, já a formação de um precipitado vermelho tijolo na presença do reagente de Dragendorff indicou a presença de alcalóides.

Para catequinas e flavonas, antocianinas, antocianidinas e flavanóides, chaconas e auronas, flavonóis e flavanonas, flavanonóis e xantonas foram identificados com a mudança de coloração a depender do meio ácido ou alcalino que foram submetidos. Esteróides e Triterpenóides foram confirmados com a presença de coloração azul seguida de verde para esteróides e parda até vermelha indicando os triterpenóides, por extração com ácido sulfúrico, clorofórmio e anidrido acético. Logo, as análises fitoquímicas realizadas, possibilitaram a identificação dos principais grupos de substâncias já referidos na literatura para essas espécies.

Em se tratando do $P$. barbatus, este possui como principal classe de metabólito secundário, os alcaloides, dentre os quais a boldina se destaca [9], para a espécie também foram encontradas na literatura a presença de fenóis, taninos, flavanóides e catequinas [12]. Na triagem química realizada com o extrato hidroalcoolico do P. barbatus foram identificados indicativos para a presença de Fenóis e Taninos, Saponinas, Alcalóides, Catequinas e Flavonas, Antocianinas, Antocianidinas e Flavanóides, Chaconas e Auronas, Flavonóis e Esteróides e Triterpenóides. Apresentando o maior número de metabólitos em relação as 3 espécies analisadas.

Para a $L$. alba foi identificado coloração específica com resultados positivos somente para Flavanonas, Flavanonóis e xantonas. Apesar de que parte dos estudos em relação a composição química da Lippia alba estejam relacionados aos seus óleos voláteis ainda é relatado na literatura a presença de taninos, flavanóides, saponinas e esteroides em extratos da planta [13, 14]. Sette-de-Souza et al. (2014) [15], ao analisarem o extrato aquoso dessa erva medicinal encontraram em sua análise fenóis, taninos e flavonoides conforme os resultados encontrados em nossa pesquisa.

Em relação a $P$. anisum foram encontrados resultados positivos para Taninos e Fenóis, Flavanonas, Flavanonóis e Xantonas. Outros estudos encontraram resultados parecidos com o dessa pesquisa, 
contudo os autores destacam a variabilidade do teor e compostos encontrados devido as formas extrativas utilizadas $[17,18]$. Esta erva medicinal está entre as principais plantas medicinais utilizadas pela população do munícipio de Lagarto conforme estudo de Caetano e colaboradores (2015) [10]. Na pesquisa, os pacientes oncológicos utilizam essa planta medicinal como tratamento alternativo para os efeitos adversos da quimioterapia, no caso da $P$. anisum no tratamento do nervosismo e desconforto abdominal causado pelos efeitos adversos da terapia.

O resultado da prospecção fitoquímica preliminar dos grupos de metabólitos secundários e constituintes de algumas dessas espécies analisadas diferem daqueles descrito na literatura, porém para algumas espécies o resultado foi similar. O que pode ser devido aos constituintes do metabolismo secundário que podem variar a depender dos fatores extrínsecos e intrínsecos, como solo, temperatura, água disponível, genética da espécie e metabolismo $[8,18]$.

Essas discrepâncias podem ocorrer quando avaliados de forma quantitativa e/ou qualitativa desses constituintes secundários e isto se deve a aspectos relacionados ao tipo de solo, tempo climático, horário de coleta do material, temperatura e reagentes químicos utilizados, são os chamados fatores edafoclimáticos, ou seja, a relação planta, solo e clima que podem sofrer interferências e variar com a espécie estudada, tendo em vista que a produção desses princípios ativos podem aumentar ou diminuir [19].

\section{Caracterização físico-química dos pós do Plectranthus barbatus, Lippia alba e Pimpinella anisum}

Determinação de perda por dessecação e teor de cinzas totais

Em relação a perda por dessecação, dentre as amostras analisadas, apenas o $P$. barbatus está dentro das especificações estabelecidas pela Farmacopeia Brasileira V ed. (2010) [9], com um valor médio de 5,69\%, na qual este resultado é de no máximo $10 \%$ (Tabela 2). Entretanto, não existem valores padronizados para as outras plantas do estudo. A preocupação com o elevado teor de umidade na amostra está relacionada principalmente com o controle microbiológico, pois o excesso de água nas amostras é prejudicial, o que favorece a atividade enzimática e a proliferação de microrganismos que poderão decompor os princípios ativos da planta, produzindo substâncias que se ingeridas podem provocar intoxicação [20], além de diminuir o valor farmacológico da droga e até anulá-lo [12].

Com base nos resultados obtidos para cinzas totais, após a calcinação da amostra do $P$. barbatus, a média do valor obtido foi de 10,09\%, um pouco acima do recomendado pela Farmacopeia Brasileira $\mathrm{V}$ ed. (2010) [9], na qual determina que o máximo de cinzas totais é de 10\%. Este valor pode ser um indicativo para presença de materiais inorgânicos não voláteis, como areia, terra ou pedras, o que demonstra problemas no momento da coleta, compra e preparo dessas amostras [21]. Os valores aceitáveis no teor de cinzas totais para $L$. alba e $P$. anisum ainda não foram descritos em nenhuma monografia e farmacopeia brasileira.

Tabela 2: Determinação de perda por dessecação e teor de cinzas totais do Plectranthus barbatus, Lippia alba e Pimpinella anisum

\begin{tabular}{ccc}
\hline Amostras & $\begin{array}{c}\text { Média da perda por } \\
\text { dessecação } \\
(\boldsymbol{\%}) \pm \mathbf{D . P}\end{array}$ & $\begin{array}{c}\text { Média do teor de cinzas } \\
\text { totais }(\%) \pm \mathbf{D . P}\end{array}$ \\
\hline Plectranthus barbatus Andrew & $5,69 \pm 0,199$ & $10,09 \pm 0,004$ \\
Lippia alba Mill & $11,35 \pm 0,218$ & $6,86 \pm 0,0037$ \\
Pimpinella anisum L. & $12,77 \pm 0,996$ & $7,27 \pm 0,0009$ \\
\hline
\end{tabular}

D.P.: Desvio Padrão

Caracterização físico-química das soluções extrativas hidroalcoólicas de Plectranthus barbatus Andrew, Lippia alba Mill e Pimpinella anisum L.

Determinação de resíduo seco, $\mathrm{pH}$ e densidade

Os valores aceitáveis para a porcentagem de resíduo seco, $\mathrm{pH}$ e densidade, para as soluções extrativas hidroaalcoólicas do $P$. barbatus, $L$. alba e $P$. anisum ainda não estão presentes na Farmacopéia Brasileira. Os resultados obtidos nas análises estão demonstrados na Tabela 3. 
Tabela 3: Determinação de resíduo seco, pH e densidade do Plectranthus barbatus, Lippia alba e Pimpinella anisum

\begin{tabular}{cccc}
\hline Amostras & $\begin{array}{c}\text { Resíduo seco } \\
(\boldsymbol{\%}) \pm \mathbf{D . P}\end{array}$ & pH & Densidade (g/ml) \\
\hline Plectranthus barbatus & $0,654 \pm 0,043$ & 5,59 & $0,925 \pm 0,004$ \\
Lippia alba & $0,082 \pm 0,088$ & 6,34 & $0,923 \pm 0,004$ \\
Pimpinella anisum & $0,029 \pm 0,06$ & 6,48 & $0,927 \pm 0,004$ \\
\hline
\end{tabular}

D.P.: Desvio Padrão

\section{CONCLUSÃO}

Para a efetivação da fitoterapia como prática segura e efetiva, o controle de qualidade é um instrumento indispensável. Durante a pesquisa observou-se a presença de locais inadequados para armazenamento e a presença de material estranho nas plantas medicinais. Nesse sentido, campanhas educativas sobre técnicas de armazenamento, detecção de contaminantes e resíduos, são vitais para diminuir o risco de possíveis intoxicações. No que diz respeito a análise fitoquímica os resultados estão em sua maioria de acordo com a literatura. $\mathrm{O}$ controle da constituição química permite a distinção entre espécies próximas evitando possíveis erros na identificação e indicação de espécies. A análise físico química desta pesquisa poderá servir de parâmetro para novos estudos visto que com relação ao $P$. anisum e o L. alba ainda não possuem dados estabelecidos pela Farmacopeia Brasileira. Entre os principais limites desta pesquisa o tamanho da amostra e a necessidade de realizar testes abrangendo outras áreas do munícipio são necessários com o objetivo de assegurar a segurança e qualidade das plantas medicinais, além de fornecer dados para a padronização e desenvolvimento de novos fármacos.

\section{REFERÊNCIAS BIBLIOGRÁFICAS}

1. Brasil. Ministério da Saúde. Agência Nacional de Vigilância Sanitária (ANVISA). Resolução da Diretoria Colegiada - $\mathrm{RDC} \mathrm{n}^{\circ} 26$, de 13 de maio de 2014. Dispõe sobre o registro de medicamentos fitoterápicos e o registro e a notificação de produtos tradicionais fitoterápicos. Disponível em: http://portal.anvisa.gov.br/wps/wcm/connect/a9e43d0044140f579b5affb9cd167b7c/rdc0026_13_05_2014.p df?MOD=AJPERES. Acesso em: 23 de maio de 2016.

2. Brasil. Ministério da Saúde. Secretaria de Ciência, Tecnologia e Insumos Estratégicos. Departamento de Assistência Farmacêutica. Política Nacional de Plantas Medicinais e Fitoterápicos / Ministério da Saúde, Secretaria de Ciência, Tecnologia e Insumos Estratégicos, Departamento de Assistência Farmacêutica. Brasília: Ministério da Saúde, 2006.

3. Souza-Moreira TM, Salgado, HRN, Pietro RCLR. O Brasil no contexto de controle de qualidade de plantas medicinais. Rev Bras Farmacogn. 2010 Jul;20(3):435-440, doi:http://dx.doi.org/10.1590/S0102695X2010000300023.

4. Brasil. Instituto Brasileiro de Geografia e Estatística (IBGE). Estimativa 2010. Brasil. Rio de Janeiro, 2013.

5. Silva JES. Diagnóstico situacional da utilização de plantas medicinais utilizadas por pacientes idosos assistidos no programa de saúde da família do município de Aracaju-SE [dissertação]. Sergipe (BR): Universidade Federal de Sergipe; 2012. 120p.

6. Atkinson R, Flint J. Accessing Hidden and Hard-to-Reach Populations: Snowball Research Strategies. In Social Research Update Department of Sociology. University of Surrey; 2001.

7. Brasil. Resolução ${ }^{\circ} 466$, de 12 de dezembro de 2012.

8. Matos FJA. Introdução a fitoquímica experimental. 3.ed. Fortaleza: Edições UFC; 2009. 150p.

9. Brasil. Farmacopeia Brasileira, v.1, 5.ed. Agência Nacional de Vigilância Sanitária. Brasília; 2010. 546p.

10. Caetano NLB, Ferreira TF, Reis MRO, Neo GGA, Carvalho AA. Plantas medicinais utilizadas pela população do município de Lagarto- SE, Brasil - ênfase em pacientes oncológicos. Rev Bras Plantas Med. 2015 17(4):749-56, doi:http://dx.doi.org/10.1590/1983-084X/14_056.

11. Junior Veiga VF, Pinto AC, Maciel MAM. Plantas medicinais: cura segura? Quím Nova. 2005 Jun;28(3):51928, doi:http://dx.doi.org/10.1590/S0100-40422005000300026.

12. Melo JG, Martins JDGR, Amorim ELC, Albuquerque UP. Qualidade de produtos à base de plantas medicinais comercializados no Brasil: castanha-da-índia (Aesculus hippocastanum L.), capim-limão 
(Cymbopogon citratus (DC.) Stapf) e centela (Centella asiatica (L.) Urban). Acta Bot Bras. 2007 Mar;21(1):27-36, doi:http://dx.doi.org/10.1590/S0102-33062007000100004.

13. Timóteo P, Karioti A, Leitão SG, Vincieri FF, Bilia AR. A validated HPLC method for the analysis of herbal teas from three chemotypes of Brazilian Lippia alba. Food Chem. 2015 Mai;175(15):366-73, doi:10.1016/j.foodchem.2014.11.129.

14. Martínez SA. Compuestos polifenólicos (extraíbles y no extraíbles) em alimentos de la dieta española: metodologia para su determinación e identificación [dissertação]. Madrid (ES): Universidade Complutense de Madrid; 2010.96 p.

15. Sette-de-Souza PH, Carneiro SER, Macedo-Costa MR, Borges SB, Medeiros AR Antibacterial activity and phytochemical screening of extracts of Lippia alba (Mill). NE Brown. Afr J Microbiol Res. 2014;8(29):278387, doi:10.5897/AJMR2014.6791.

16. Yacoub OSM, Embarek A, Abderahim K, Abdelmoula EO, Bouchra B, Ali O, Aboubaker EH, Omar A, Abdelhalim M. Chemical Composition and Zootechnical Effects of Essential Oil of Fennel (Foeniculum Vulgare Mill) and Anise (Pimpinella Anisum L.) on Turkey. Journal of World's Poultry Research 2015;5(4):90-97.

17. Shojaii A, Fard MA. Review of pharmacological properties and chemical constituents of Pimpinella anisum. International Scholarly Research Network Pharmaceutics 2012, doi:10.5402/2012/510795.

18. Miranda CASF, Cardoso MdasG, Batista LR, Rodrigues LMA, Figueiredo AC da Silva. Óleos essenciais de folhas de diversas espécies: propriedades antioxidantes e antibacterianas no crescimento espécies patogênicas. Rev Ciênc Agron. 2016 Mar;47(1):213-20, doi:https://dx.doi.org/10.5935/18066690.20160025 .

19. Gobbo-Neto L, Lopes NP. Plantas medicinais: fatores de influência no conteúdo de metabólitos secundários. Quim Nova. 2007;30(2):374-81.

20. Nascimento VT, Lacerda EU, Melo JG, Lima CSA, Amorim ELC, Albuquerque UP. Controle de qualidade de produtos à base de plantas medicinais comercializados na cidade de recife-PE: erva-doce (Pimpinella anisum L.), quebra-pedra (Phyllanthus ssp.), espinheira-santa (Maytenus ilicifolia Mart.) e camomila (Matricaria recutita L.). Rev Bras Pl Med. 2005;7(3):56-64.

21. Almeida AA, Ming TD, Corrêa CL, Lavinas T. Verificação da qualidade do fitoterápico sene e boldo-dochile comercializados na região de campinas. Rev Ciênc Med 2005 Jun;14(3):279-85. 